\title{
Localized prostate cancer is rarely a fatal disease
}

\author{
Peter T Scardino
}

A medical oncology colleague recently approached me about a new drug regimen for prostate cancer that, if used in combination with radical prostatectomy (RP), could substantially improve survival in men at high risk of eventually dying from their cancer. "Of all the men that you operate on," he asked, "which are at high risk of eventually dying of the disease?"

His question followed the publication of our recent study, which argued that "high risk" is a term too loosely applied to localized prostate cancer. More than half of men considered to have high-risk cancer, by any of the commonly used definitions, are cured by surgery alone (Yossepowitch $\mathrm{O}$ et al. [2007] J Urol 178: 493-499), and few are likely to die of their cancer within 10 years. Digging deeper into our large database of patients treated since 1983, we found that the cancer recurred in about one in every four men, while death from cancer within 15 years occurred in only one in eight men. Even when the cancer recurred following RP, the risk of dying from cancer within 15 years of the first rise in PSA level was only about 30\% (Bianco F et al. [2005] Urology 66 [Suppl 5A]: 83-94).

With widespread screening for prostate cancer in the US, lower PSA thresholds for biopsy and increasing numbers of cores taken per biopsy session, there has been a marked shift toward detection of prostate cancer at an earlier stage (Cagiannos I et al. [2004] J Urol 171: 692-696). We, therefore, examined the features of cancer in our patients treated since 1998, when the favorable stage shift was largely complete. In this group, we calculated

\section{Knowing \\ the risk of \\ morbidity... \\ from \\ treatment, is it \\ reasonable for \\ us to continue \\ to treat low- \\ risk prostate \\ tumors with \\ radical surgery \\ or radiation \\ therapy?}

PT Scardino is

Editor-in-Chief of Nature Clinical

Practice Urology.

\section{Competing interests}

The author declared no

competing interests.

www.nature.com/clinicalpractice doi:10.1038/ncpuro1013 that fewer than 1 in 1,000 men would have a $>30 \%$ risk of death from cancer at 15 years after diagnosis. At the end of this exercise, I reported back to my medical oncology colleague that I could not identify any men currently being treated with RP for localized prostate cancer whose risk of dying from cancer within 15 years was sufficiently high to justify adjuvant systemic therapy.

Granted, all the men in the series we analyzed were treated with RP; none were managed by active surveillance. But their extraordinarily favorable prognosis raises the question of whether the subset with low-risk cancer should be exposed to the risk of immediate therapy. If prostate cancer detected in a heavily screened population poses so little risk of death, should it properly be termed "cancer"? Klotz reported a 99\% 8-year disease-specific survival rate in a large group of men with low-risk and intermediate-risk cancers managed expectantly - that is, without initial therapy but with radical treatment only if the cancer later progressed (Klotz L [2005] $J$ Clin Oncol 23: 8165-8169). We found the same outcome in a smaller series (Patel MI et al. [2004] J Urol 171: 1520-1524). Can patients diagnosed with localized prostate cancer comprehend how little risk their cancer poses to their long-term survival? Knowing the risk of morbidity, and even mortality, from treatment, is it reasonable for us to continue to treat low-risk prostate tumors with radical surgery or radiation therapy? One wonders how much longer the era of radical therapy for small, early prostate cancers can last. 